\title{
Article \\ Proteomic Determination of Low-Molecular-Weight Glutenin Subunit Composition in Aroona Near-Isogenic Lines and Standard Wheat Cultivars
}

\author{
Kyoungwon Cho ${ }^{1}$ (D), You-Ran Jang ${ }^{2}$, Sun-Hyung Lim $^{3}$, Susan B. Altenbach ${ }^{4}$, Yong Q. Gu ${ }^{4}$, \\ Annamaria Simon-Buss ${ }^{4,5}$ and Jong-Yeol Lee ${ }^{2, *}$
}

1 Department of Biotechnology, College of Agriculture and Life Sciences, Chonnam National University, Gwangju 61186, Korea; kw.cho253@gmail.com

2 National Institute of Agricultural Science, RDA, Jeonju 54874, Korea; jang6122@korea.kr

3 Division of Horticultural Biotechnology, Hankyong National University, Anseong 17579, Korea; limsh2@hknu.ac.kr

4 USDA-ARS, Western Regional Research Center, 800 Buchanan Street, Albany, CA 94710, USA; susan.altenbach@usda.gov (S.B.A.); yong.gu@usda.gov (Y.Q.G.); Anna.Simon-Buss@chemie.uni-hamburg.de (A.S.-B.)

5 Hamburg School of Food Science, Institute of Food Chemistry, University of Hamburg, 20146 Hamburg, Germany

* Correspondence: jy0820@korea.kr; Tel.: +82-62-238-4616

Citation: Cho, K.; Jang, Y.-R.; Lim, S.-H.; Altenbach, S.B.; Gu, Y.Q.; Simon-Buss, A.; Lee, J.-Y. Proteomic Determination of Low-MolecularWeight Glutenin Subunit

Composition in Aroona

Near-Isogenic Lines and Standard Wheat Cultivars. Int. J. Mol. Sci. 2021, 22, 7709. https://doi.org/10.3390/ ijms22147709

Academic Editor: Pedro Martínez-Gómez

Received: 24 June 2021

Accepted: 15 July 2021

Published: 19 July 2021

Publisher's Note: MDPI stays neutral with regard to jurisdictional claims in published maps and institutional affiliations.

Copyright: (c) 2021 by the authors. Licensee MDPI, Basel, Switzerland. This article is an open access article distributed under the terms and conditions of the Creative Commons Attribution (CC BY) license (https:// creativecommons.org/licenses/by/ $4.0 /)$.

\begin{abstract}
The low-molecular weight glutenin subunit (LMW-GS) composition of wheat (Triticum aestivum) flour has important effects on end-use quality. However, assessing the contributions of each LMW-GS to flour quality remains challenging because of the complex LMW-GS composition and allelic variation among wheat cultivars. Therefore, accurate and reliable determination of LMW-GS alleles in germplasm remains an important challenge for wheat breeding. In this study, we used an optimized reversed-phase HPLC method and proteomics approach comprising 2-D gels coupled with liquid chromatography-tandem mass spectrometry (MS/MS) to discriminate individual LMW-GSs corresponding to alleles encoded by the Glu-A3, Glu-B3, and Glu-D3 loci in the 'Aroona' cultivar and 12 'Aroona' near-isogenic lines (ARILs), which contain unique LMW-GS alleles in the same genetic background. The LMW-GS separation patterns for 'Aroona' and ARILs on chromatograms and 2-D gels were consistent with those from a set of 10 standard wheat cultivars for Glu-3. Furthermore, 12 previously uncharacterized spots in 'Aroona' and ARILs were excised from 2-D gels, digested with chymotrypsin, and subjected to MS/MS. We identified their gene haplotypes and created a 2-D gel map of LMW-GS alleles in the germplasm for breeding and screening for desirable LMW-GS alleles for wheat quality improvement.
\end{abstract}

Keywords: LMW-GS; proteomic analysis; RP-HPLC; Aroona near-isogenic lines; standard wheat cultivars

\section{Introduction}

Gluten proteins comprise $70-80 \%$ of the total wheat flour protein; give wheat dough its unique viscoelastic properties; and make it possible to produce bread, pasta, noodles, and other products [1,2]. Gluten proteins can be separated on the basis of their solubility into monomeric gliadins that dissolve in $60-80 \%$ alcohol and polymeric glutenins that are weakly acidic and alkaline-soluble [3,4]. Glutenins consist of high-molecular-weight glutenin subunits (HMW-GSs; 70-90 kDa) and low-molecular-weight glutenin subunits (LMW-GSs; 20-45 kDa) that are polymerized by inter-molecular disulfide bonds [5]. HMWGSs are encoded by genes at the Glu-1 loci (Glu-A1, Glu-B1, and Glu-D1) on the long arms of homoeologous group 1 chromosomes, with three to five active genes in most bread wheat cultivars. LMW-GSs are encoded by genes at the Glu-3 loci (Glu-A3, Glu-B3, and 
Glu-D3) on the short arms of homoeologous group 1 chromosomes, with the gene copy number varying from 10 to 40 [6,7]. Genetic differences in HMW-GSs and LMW-GSs among wheat cultivars affect the physical properties of dough and determine their end-use qualities [8,9]. Allelic variants of LMW-GSs affect dough strength and extensibility and are far more complicated than those of HMW-GSs that correlate with dough elasticity [10-14].

Glutens can be further divided into the A-, B-, C--, and D-groups according to their mobility in SDS-PAGE based on molecular weight. HMW-GSs and LMW-GSs make up the A- and B-groups, respectively. The C-group contains $\alpha / \beta$ - and $\gamma$-gliadins and some LMWGSs, while the D-group comprises mostly $\omega$-gliadins and some LMW-GSs [9], indicating that LMW-GSs in the C- and D-groups overlap with gliadins in SDS-PAGE. Most genes encoding LMW-GSs in groups B, C, and D are located close to the Gli-1 loci encoding $\gamma$ and $\omega$-gliadin on the short arms of group 1 chromosomes [15]. Some LMW-GSs in the C-group are located near the Gli-2 loci encoding $\alpha / \beta$-gliadin on the short arms of group 6 chromosomes [16]. The close location of Gli-1/-2 encoding gliadin and Glu-3 encoding C/D-group LMW-GSs and their frequent recombination make analyzing the gene structure and function of LMW-GSs difficult $[17,18]$.

Many researchers have studied individual LMW-GS alleles due to their importance in wheat breeding. The allelic composition of LMW-GS has been analyzed by PCR [19], SDS-PAGE [20,21], two-dimensional gel electrophoresis (2-DGE) [22,23], and reversed phase-ultra high-performance liquid chromatography (RP-UHPLC) $[20,21,24]$. In hexaploid wheat cultivars, six $(a, b, c, d, e$, and $f)$, nine $(a, b, c, d, e, f, g, h$, and $i)$, and five $(a, b, c, d$, and e) allelic forms at the Glu-A3, Glu-B3, and Glu-D3 loci, respectively, have been reported [25]. Four, three, and seven genes were identified at the Glu-A3d, Glu-B3b, and Glu-D3c LMW-GS alleles, respectively, in the Chinese wheat cultivar 'Xiaoyan 54' [26]. Using proteomic analysis coupled with 2-DGE and tandem mass spectrometry (MS/MS), one, two, and five LMW-GSs were identified at the Glu-A3c, Glu-B3h, and Glu-D3a in the Korean cultivars 'Jokyung' [27] and 'Keumkang' [28]. In the Australian and New Zealand cultivars, Glu-3 alleles have been ranked according to dough resistance [25,29], thereby determining the best combinations of Glu-3 alleles for dough quality [11] and ranking each allele for dough strength by analyzing the relationship between the allelic variation of $\mathrm{Glu}-3$ and bread wheat quality [30]. Additionally, Zhang et al. (2012) [19] and Rasheed et al. (2014) [31] used near-isogenic lines from the 'Aroona' cultivar to rank each allele at the Glu-3 loci for dough strength and extensibility, providing information for predicting dough-processing qualities based on the composition of LMW-GS alleles.

Despite efforts to accurately and reliably determine LMW-GS alleles in wheat germplasm, the contributions of LMW-GS alleles to quality have been difficult to assess. This is a result of a lack of efficient techniques for isolating individual LMW-GS genes from the multiple highly similar LMW-GS genes within a cultivar and the allelic variation between cultivars. To identify LMW-GSs corresponding to different alleles encoded by the Glu-A3, Glu-B3, and Glu-D3 loci, we fractionated glutenins from 'Aroona', a set of 12 'Aroona' near-isogenic lines (ARILs) containing unique LMW-GS alleles in the same genetic background, and a set of 10 standard wheat cultivars. Glutenins were separated using optimized methods for RP-HPLC and 2-DGE combined with LC-MS/MS. We compared the patterns of separated LMW-GSs on chromatograms and 2-D gels and identified peaks and protein spots corresponding to each LMW-GS allele through comparative analysis among ARILs and standard wheat cultivars. The results will be used to identify LMW-GS alleles in germplasm prior to breeding and to screen for desirable LMW-GS alleles for wheat quality improvement.

\section{Results}

\subsection{Identification of LMW-GS Alleles Using RP-HPLC}

To determine the allelic composition of low-molecular-weight glutenin subunits (LMWGSs) in 'Aroona' and 12 'Aroona' near-isogenic lines (ARILs; Table 1), we extracted their glutenin fractions and analyzed them by RP-HPLC. The separation of LMW-GSs was optimized under the following conditions: linear gradient from 25 to $45 \%$ solvent B and 
$0.8 \mathrm{~mL} / \mathrm{min}$ flow rate for $50 \mathrm{~min}$ at $65^{\circ} \mathrm{C}$. We observed five HMW-GS peaks between 5 and $20 \mathrm{~min}$, and LMW-GS and gliadin peaks were detected between 20 and $45 \mathrm{~min}$ (Figure 1). HMW-GS alleles in 'Aroona' comprise Ax1 at the Glu-A1 locus, Bx7 and By9 at the Glu-B1 locus, and Dx2 and Dy12 at the Glu-D1 locus [32], which can be identified as peaks in RP-HPLC based on hydrophobicity [33]. We identified the peaks corresponding to each LMW-GS encoded at Glu-3 loci by comparing LC peak patterns in 'Aroona' with those in 12 ARILs: six alleles $(a, b, c, d, e$, and $f)$ at $G l u-A 3$, six $(a, b, c, d, g$, and $h)$ at $G l u-B 3$, and three ( $b$, $c$, and $f$ ) at Glu-D3 (Figure 1).

Table 1. Allelic compositions of LMW-GS at the Glu-A3, Glu-B3, and Glu-D3 loci in 'Aroona' and 'Aroona' near isogenic lines (ARILs).

\begin{tabular}{ccccc}
\hline Line & Glu-A3 & Glu-B3 & Glu-D3 & Donor Parent \\
\hline Aroona & $c$ & $b$ & $c$ & Aroona \\
ARIL 15-4 & $a$ & $b$ & $c$ & Chinese spring \\
ARIL 16-1 & $b$ & $b$ & $c$ & Gabo \\
ARIL 18-5 & $d$ & $b$ & $c$ & Orca \\
ARIL 19-2 & $e$ & $b$ & $c$ & Lerma Rojo \\
ARIL 20-1 & $f$ & $b$ & $c$ & Bungulla \\
ARIL 21-2 & $c$ & $a$ & $c$ & Chinese spring \\
ARIL 23-4 & $c$ & $c$ & $c$ & Halberd \\
ARIL 24-3 & $c$ & $d$ & $c$ & Orca \\
ARIL 27-6 & $c$ & $h$ & $c$ & Millewa \\
ARIL 28-4 & $c$ & $b$ & $b$ & Sonalika \\
ARIL 36-2 & $c$ & $b$ & $f$ & Bungulla \\
ARIL 35-1 & $c$ & & & India 115 \\
\hline
\end{tabular}

To identify LMW-GS alleles at the Glu-A3 locus, we compared LMW-GS peaks in lines ARIL 15-4 (Glu-A3a), ARIL 16-1 (Glu-A3b), 'Aroona' (Glu-A3c), ARIL 18-5 (Glu-A3d), ARIL 19-2 (Glu-A3e), and ARIL 20-1 (Glu-A3f). Peaks that were not common to all Glu-A3 allelic lines are marked with red arrowheads in Figure 1A. We detected two LMW-GS peaks in ARIL 15-4 (Glu-A3a), ARIL 16-1 (Glu-A3b), and 'Aroona' (Glu-A3c) that could not be distinguished by RP-HPLC because they had the same retention time. However, we detected five, three, and two unique peaks in ARIL 18-5 (Glu-A3d), ARIL 19-2 (Glu-A3e), and ARIL 20-1 (Glu-A3f), respectively. These results indicate that it is difficult to discriminate the Glu-A3a, $-A 3 b$, and $-A 3 c$ alleles by RP-HPLC but easy to distinguish the Glu-A3d,-A3e, and $-A 3 f$ alleles.

We analyzed the Glu-B3 alleles in 'Aroona' (Glu-B3b) and ARIL 21-2 (Glu-B3a), ARIL 23-4 (Glu-B3c), ARIL 24-3 (Glu-B3d), ARIL 27-6 (Glu-B3g), and ARIL 28-4 (Glu-B3h). After excluding peaks common to all $G l u-B 3$ allelic lines, the remaining peaks were marked with blue arrows as shown in Figure 1B. We detected two or four LMW-GS peaks between 25 and $45 \mathrm{~min}$. Two unique LMW-GS peaks were eluted at similar retention times in 'Aroona' (Glu-B3b) and ARIL 23-4 (Glu-B3c). Compared to LMW-GS peak patterns in 'Aroona' (Glu$B 3 b$ ), four distinct peaks were detected at different retention times, respectively, in ARIL 21-2 (Glu-B3a), ARIL 24-3 (Glu-B3d), ARIL 28-4 (Glu-B3h), and ARIL 27-6 (Glu-B3g). One additional peak (black arrow) eluted between 5 and 25 min was observed in ARIL 27-6 $(G l u-B 3 g)$ and in standard wheat cultivar, 'Glenlea' (Glu-B3g) (Figure S1).

For LMW-GS alleles at the Glu-D3 locus, we analyzed 'Aroona' (Glu-D3c), ARIL 36-2 (Glu-D3b), and ARIL 35-1 (Glu-D3f), as shown in Figure 1C. Comparing LMW-GS peaks among the three lines, we detected three, four, and four unique peaks (green arrowheads in Figure 1C) in 'Aroona' (Glu-D3c), ARIL 36-2 (Glu-D3b), and ARIL 35-1 (Glu-D3f), respectively. LMW-GS peaks in ARIL 36-2 (Glu-D3b) and ARIL 35-1 (Glu-D3f) showed similar retention times, indicating that it is difficult to discriminate the $G l u-D 3 b$ and $-D 3 f$ alleles using RP-HPLC. 
(A)
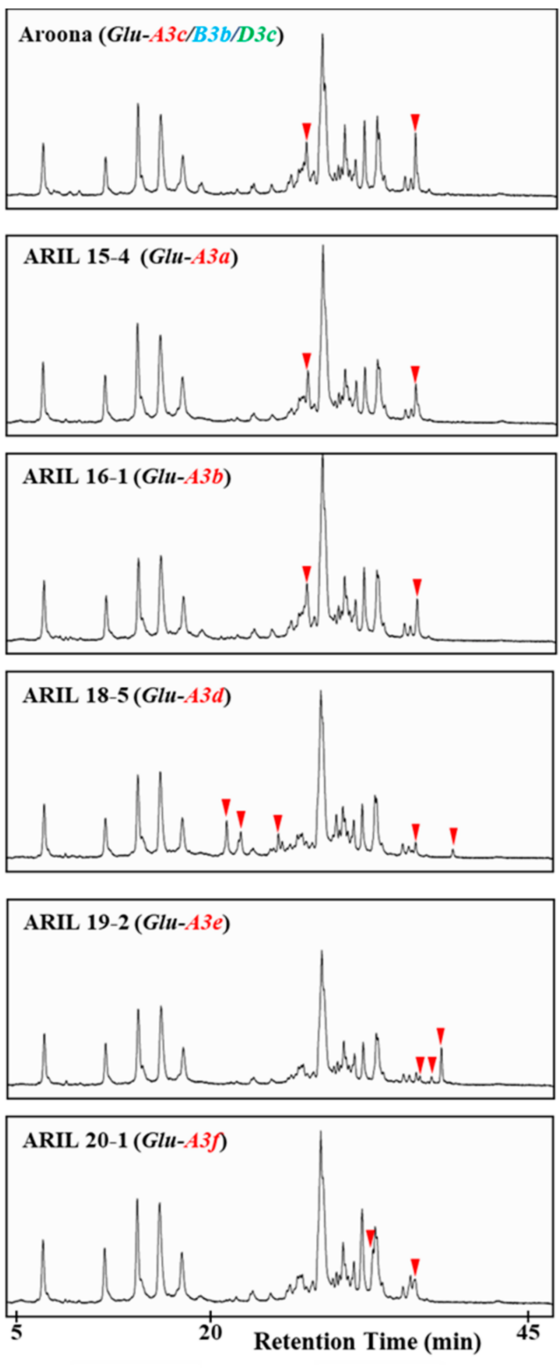

(B)
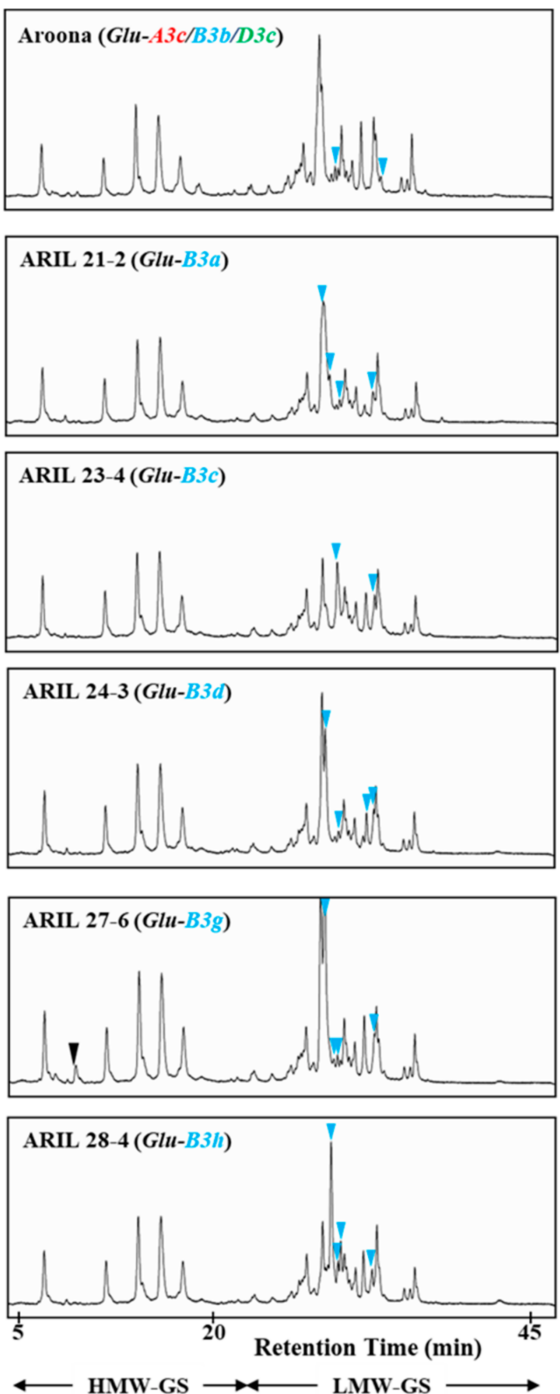

(C)
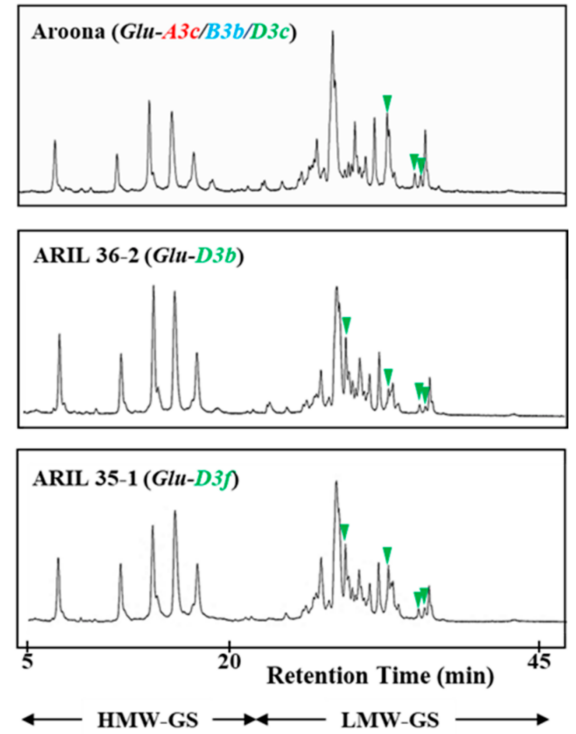

Figure 1. RP-HPLC analysis of LMW-GS fractions in 'Aroona' and ARILs. Peaks corresponding to each LMW-GS allele encoded by Glu-A3 (A), Glu-B3 (B), and Glu-C3 (C) are indicated by red, light blue, and green arrowheads, respectively.

To confirm the accuracy of our LMW-GS identification in 'Aroona' and the 12 ARILs, we compared peak patterns of LMW-GSs extracted from 10 standard wheat cultivars (Table S1). Peaks corresponding to LMW-GS alleles at Glu-A3, Glu-B3, and Glu-D3 loci are marked with red, blue, and green arrowheads, respectively, in Figure S1. We detected two unique peaks for Glu-A3a and four unique peaks for Glu-B3a in 'Chinese Spring' $(G l u-A 3 a / G l u-B 3 a)$. LMW-GS peaks in 'Gabo' with Glu-A3b, Glu-B3b, and Glu-D3b alleles matched two, two, and four peaks observed in ARIL 16-1 (Glu-A3b), 'Aroona' (Glu-B3b), and ARIL 36-2 (Glu-D3b), respectively. Two LMW-GS peaks detected in 'Aroona' (Glu$A 3 c$ ) and four detected in ARIL 35-1 (Glu-D3f) were also detected in 'Cheyenne' (Glu-A3c and Glu-D3f). Five LMW-GS peaks in ARIL 18-5 (Glu-A3d) and four peaks in ARIL 24-3 $(G l u-B 3 d)$ were also observed in the 'Orca' cultivar (Glu-A3d and Glu-B3d). Moreover, three LMW-GS peaks in ARIL 19-2 (Glu-A3e), two in ARIL 20-1 (Glu-A3f), two in ARIL 23-4 (Glu-B3c), four in ARIL 27-6 (Glu-B3g), four in ARIL 28-4 (Glu-B3h), and three in 'Aroona' $(G l u-D 3 c)$ were detected in their standard cultivars 'Neepawa' (Glu-A3e), 'Clement' (Glu$A 3 f)$, 'Halberd' (Glu-B3c), 'Glenlea' (Glu-B3g), 'Suneca' (Glu-B3h), and 'Insignia' (Glu-D3c), respectively (Figure S1). 


\subsection{Comparison of LMW-GSs among 'Aroona' and Its Near-Isogenic Lines Using 2-DGE}

Spot patterns for LMW-GS alleles in 2-DGE were analyzed from 'Aroona' and its 12 ARILs. Previously, LMW-GS alleles encoded at the Glu-3 locus were successfully identified in 32 Korean wheat cultivars and 11 standard wheat cultivars [23]. These results facilitated the identification of LMW-GSs alleles in 'Aroona' and its ARILs.

For the identification of Glu-A3 alleles, LMW-GS fractions were extracted from 'Aroona' (Glu-A3c), ARIL 15-4 (Glu-A3a), ARIL 16-1 (Glu-A3b), ARIL 18-5 (Glu-A3d), ARIL 19-2 (Glu$A 3 e$ ), and ARIL 20-1 (Glu-A3f) and separated on 2-D gels (Figure 2A). Four spots (shown within a black square in Figure 2) at about $43 \mathrm{kDa}$ and $9.2 \mathrm{pI}$ on each gel were used as a reference to compare gel patterns as reported in a previous study [23]. Based on previous 2-DGE results for the LMW-GS encoded by the Glu-A3c allele in Korean wheat cultivars 'Keumkang' [28] and 'Jokyung' [27], we defined the LMW-GS spot (red circle) corresponding to the Glu-A3c allele in 'Aroona'. LMW-GSs from ARIL 15-4 (Glu-A3a), ARIL 16-1 (Glu-A3b), and 'Aroona' (Glu-A3c) showed the same patterns in 2-DGE, indicating the difficulty in discriminating the Glu-A3a,-A3b, and $-A 3 c$ alleles using 2-DGE as well as RP-HPLC. Four and one unique protein spots were detected in 2-DGE of ARIL 18-5 (Glu-A3d) and ARIL 20-1 (Glu-A3f), respectively, but no distinct spots were observed for ARIL 19-2 (Glu-A3e), as shown in Figure 2A.

For the analysis of Glu-B3 alleles using 2-DGE, we extracted LMW-GSs from 'Aroona' (Glu-B3b), ARIL 21-2 (Glu-B3a), ARIL 23-4 (Glu-B3c), ARIL 24-3 (Glu-B3d), ARIL 27-6 (Glu$B 3 g)$, and ARIL 28-4 (Glu-B3h). Figure 2B shows two proteins (light blue arrows) within the black square at about $43 \mathrm{kDa}$ and $9.2 \mathrm{pI}$ that were commonly detected in 'Aroona' and all the ARILs. Four spots (light blue circles) corresponding to proteins of about $43 \mathrm{kDa}$ with pIs between 9.2 and 10 pI were detected in 'Aroona' (Glu-B3b) and ARIL 27-6 (Glu-B3g). Only two spots in this region were detected in ARIL 21-2 (Glu-B3a), while none were detected in ARIL 23-4 (Glu-B3c), ARIL 24-3 (Glu-B3d), or ARIL 28-4 (Glu-B3h). Instead, two, three, and four proteins (light blue circles) with higher molecular weights than those in 'Aroona' (Glu-B3b) were detected in ARIL 23-4 (Glu-B3c), ARIL 24-3 (Glu-B3d), and ARIL 28-4 (Glu-B3h), respectively (Figure 2B). Moreover, compared to those in 'Aroona' (Glu-B3b), ARIL 27-6 (Glu-B3g) had three additional proteins (spots 9, 10, and 11) on the more basic side.

To identify LMW-GSs at the Glu-D3 locus, we analyzed 'Aroona' (Glu-3Dc), ARIL 36-2 (Glu-D3b), and ARIL 35-1 (Glu-D3f). Two proteins (green arrows within the black square in Figure 2C) derived from Glu-3Dc in 'Aroona' were detected in all lines. Six LMW-GSs (green circles) in 'Aroona' (Glu-3Dc) were not detected in ARIL 36-2 (Glu-D3b) or ARIL 35-1 (Glu-D3f). Three unique proteins were observed in ARIL 36-2 (Glu-D3b) and ARIL 35-1 (Glu-D3f), indicating that LMW-GSs encoded by Glu-D3b and Glu-D3f alleles might be highly conserved, making it difficult to distinguish them using 2-DGE.

We previously performed LMW-GS allelic analysis in 11 standard wheat cultivars using 2-DGE [23]. Based on this 2-DGE data and the LMW-GS allelic composition of 10 standard wheat cultivars (Table S1), we reconfirmed the LMW-GS alleles of 'Aroona' and the 12 ARILs (Figure S2). One LMW-GS (red circle) detected in 'Chinese Spring' (Glu-A3a), 'Gabo' (Glu-A3b), and 'Cheyenne' (Glu-A3c) was also observed at a similar gel position among LMW-GSs from ARIL 15-4 (Glu-A3a), ARIL 16-1 (Glu-A3b), and 'Aroona' (Glu-A3c), respectively (Figure 2A). Similar to ARIL 18-5 (Glu-A3d), ARIL 20-1 (Glu-A3f), and ARIL 19-2 (Glu-A3e), respectively, four, one, and no LMW-GSs were detected in 'Orca' (Glu-A3d), 'Clement' (Glu-A3f), and 'Neepawa' (Glu-A3e), respectively. 
(A)
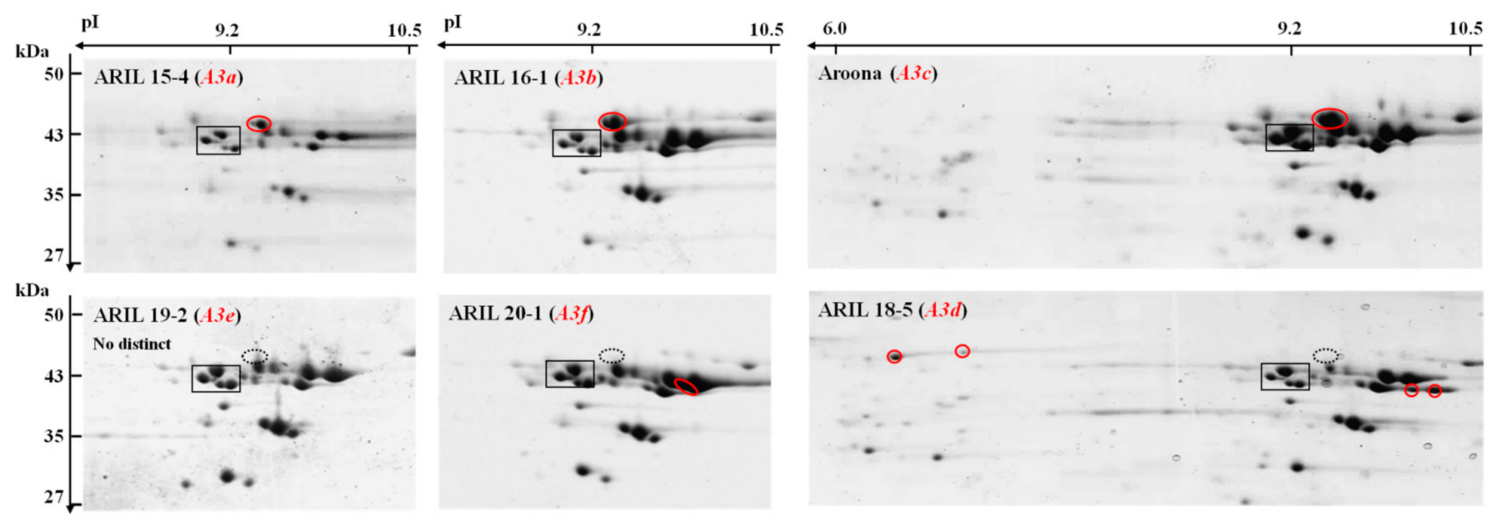

(B)
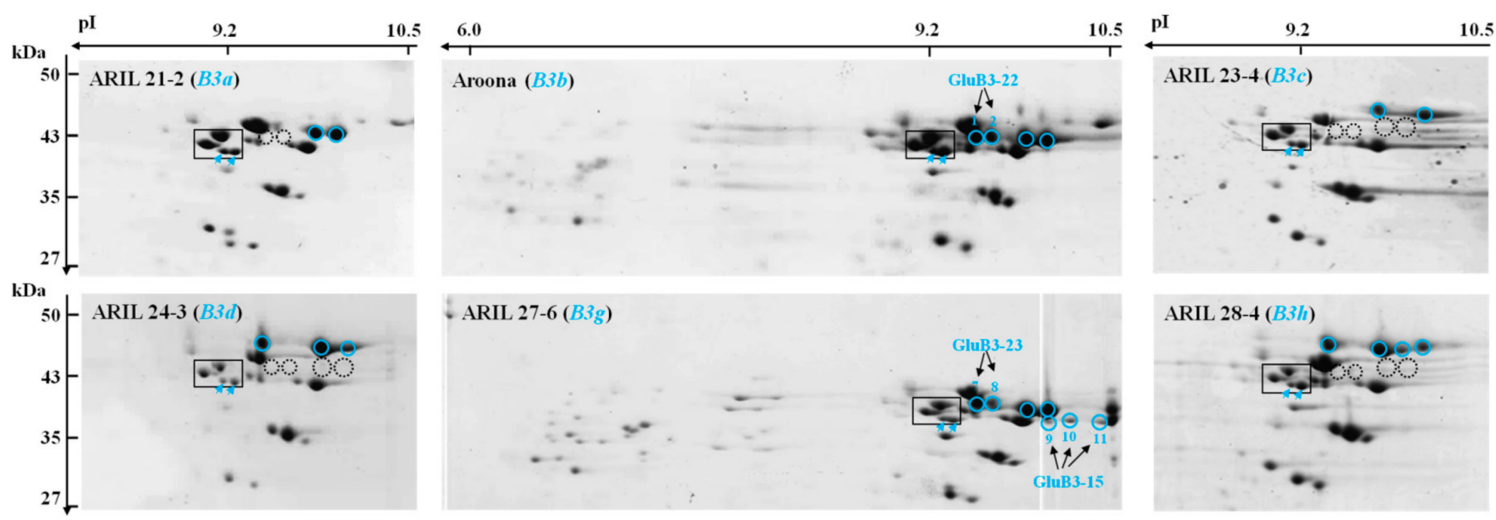

(C)
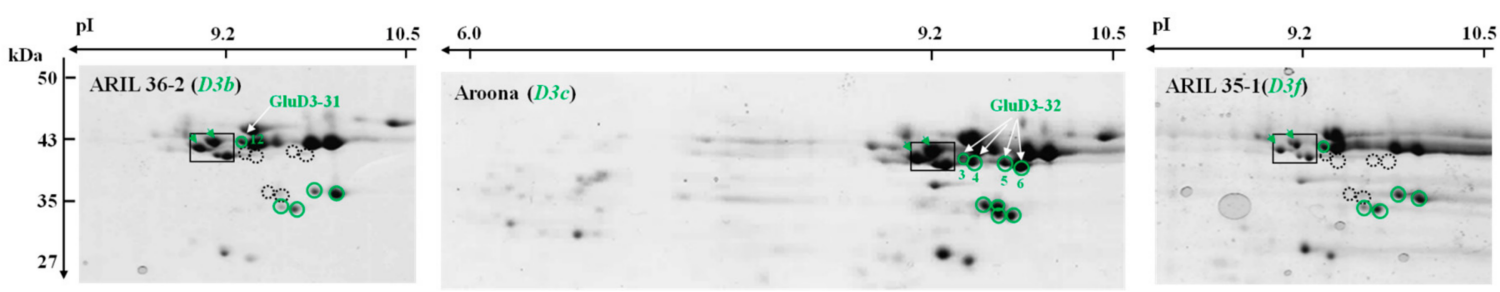

Figure 2. Two-dimensional gel electrophoresis (2-DGE) analysis of LMW-GSs from 'Aroona' and its ARILs. Protein spots corresponding to each LMW-GS allele encoded by Glu-A3 (A), Glu-B3 (B), and Glu-C3 (C) are indicated by red, light blue, and green arrows, respectively. Dashed black circles indicate the absence of spots detected in 'Aroona'. Numbered spots were identified using LC-MS/MS.

We analyzed LMW-GS alleles at the Glu-B3 locus in six standard cultivars: 'Chinese Spring' (Glu-B3a), 'Gabo' (Glu-B3b), 'Halberd' (Glu-B3c), 'Orca' (Glu-B3d), 'Suneca' (Glu$B 3 b)$, and 'Glenlea' (Glu-B3g). Unique LMW-GS spots in 2-DGE of ARIL 21-2 (Glu-B3a), 'Aroona' (Glu-B3b), ARIL 23-4 (Glu-B3c), ARIL 24-3 (Glu-B3d), ARIL 27-6 (Glu-B3g), and ARIL 28-4 (Glu-B3h) were similarly positioned on 2-DGE gels of the corresponding standard cultivars, respectively (Supplementary Figure S2). Moreover, for Glu-D3 alleles, three LMWGSs in ARIL 36-2 (Glu-D3b) and six in 'Aroona' (Glu-D3c) were also detected in 'Gabo' $(G l u-D 3 b)$ and 'Insignia' (Glu-D3c), respectively; however, one of three spots detected in 2-DGE of ARIL 35-1 (Glu-D3f) was not observed in the 2-DGE of 'Cheyenne' (Glu-D3f).

\subsection{Identification of LMW-GSs Using LC-MS/MS Analysis}

We found that the annotation of 24 LMW-GS spots in the 2-DGE of 'Aroona', ARILs, and standard wheat cultivars did not match that from our previous study on LMW-GS alleles in standard wheat cultivars and Korean wheat cultivars [23]. To identify mismatched spots, they were excised from stained 2-DGE gels, digested in-gel with either chymotrypsin, 
thermolysin, or trypsin ('Aroona' and ARILS) or only chymotrypsin (standard and Korean cultivars) and then subjected to tandem mass spectrometry (LC-MS/MS) (Table 2 and Supplementary Table S2). For LMW-GS alleles encoded at the Glu-3B locus, we identified two proteins (spots 1 and 2) in 'Aroona' (Glu-B3b), two (spots 22 and 23) in 'Gabo' (Glu-B3b), five (spots 7, 8, 9, 10, and 11) in ARIL 27-6 (Glu-B3g), and five (spots 24, 25, 26, 27, and 28) in 'Glenlea' (Glu-B3g). Spot 1 from the 2-DGE of 'Aroona' (Glu-B3b) was an LMW-GS encoded by GluB3-23, while spot 2 for 'Aroona' (Glu-B3b) and spots 22/23 for 'Gabo' (Glu-B3b) were LMW-GSs encoded by GluB3-22. In the case of LMW-GS alleles encoded by Glu-B3g, we identified spot 7/8 for ARIL 27-6 (Glu-B3g) and spots 24/25 for 'Glenlea' (Glu-B3g) as LMW-GSs encoded by GluB3-23, and spot 10 for ARIL 27-6 (Glu-B3g) and spots 26/27/28 for 'Glenlea' (Glu-B3g) as LMW-GSs encoded by GluB3-15. Moreover, spots 9 and 11 in 2-DGE of ARIL 27-6 (Glu-B3g) were identified as LMW-GSs encoded by GluB3-16, and -51, respectively.

Table 2. Identification of LMW-GSs at the Glu-A3, Glu-B3, and Glu-D3 loci in 'Aroona' and 'Aroona' near isogenic lines (ARILs).

\begin{tabular}{|c|c|c|c|c|c|c|c|c|}
\hline \multirow{2}{*}{ \# Spot } & \multirow{2}{*}{ Cultivars } & \multirow{2}{*}{ Gene } & \multirow{2}{*}{$\begin{array}{c}\text { MS/MS } \\
\text { Identification }\end{array}$} & \multirow{2}{*}{$\begin{array}{l}\text { a Gene } \\
\text { Haplotype }\end{array}$} & \multirow{2}{*}{$\begin{array}{c}\mathrm{N}- \\
\text { Terminal } \\
\text { Sequence }\end{array}$} & \multirow{2}{*}{$\begin{array}{c}\# \\
\text { A.A }\end{array}$} & \multicolumn{2}{|c|}{$\begin{array}{c}\text { b Putative Corresponding } \\
\text { Genes }\end{array}$} \\
\hline & & & & & & & $\begin{array}{l}\text { Accession No. } \\
\text { (Identity) }\end{array}$ & Gene \\
\hline 1 & AROONA & Glu-B3b & ACA63873 & GluB3-22 & MENSHIP & 349 & EU369721 (100\%) & B3-621a \\
\hline 2 & AROONA & Glu-B3b & ACA63873 & GluB3-22 & MENSHIP & 349 & EU369721 (100\%) & B3-621a \\
\hline 3 & AROONA & Glu-D3c & ABC 84367 & GluD3-32 & IENSHIP & 334 & FJ755316 (100\%) & D3-578a \\
\hline 4 & AROONA & Glu-D3c & ABC84367 & GluD3-32 & IENSHIP & 334 & FJ755316 (100\%) & D3-578a \\
\hline 5 & AROONA & $G l u-D 3 c$ & ABC84367 & GluD3-32 & IENSHIP & 334 & FJ755316 (100\%) & D3-578a \\
\hline 6 & AROONA & $G l u-D 3 c$ & АBC 84367 & GluD3-32 & IENSHIP & 334 & FJ755316 (100\%) & D3-578a \\
\hline 7 & ARIL 27-6 & Glu-B3g & ACA63857 & GluB3-23 & MENSHIP & 349 & EU369705 (100\%) & B3-621b \\
\hline 8 & ARIL 27-6 & Glu-B3g & ACA63857 & GluB3-23 & MENSHIP & 349 & EU369705 (100\%) & $B 3-621 b$ \\
\hline 9 & ARIL 27-6 & Glu-B3g & ABY58126 & GluB3-15 & MENSHIP & 323 & EU369703 (100\%) & B3-544 \\
\hline 10 & ARIL 27-6 & Glu-B3g & ABY58126 & GluB3-15 & MENSHIP & 323 & EU369703 (100\%) & B3-544 \\
\hline 11 & ARIL 27-6 & Glu-B3g & ACZ59817 & - & MENSHIP & 324 & EU369703 (100\%) & B3-544 \\
\hline 12 & ARIL 36-2 & $G l u-D 3 b$ & ABC84366 & GluD3-31 & MENSHIP & 344 & JX878006 (100\%) & D3-578b \\
\hline
\end{tabular}

a LMW-GS genes identified from Glu-B3 [34] and Glu-D3 [35] b LMW-GS genes isolated from 'Aroona' and ARILs [19,36].

We used one LMW-GS (spot 12) in ARIL 36-2 (Glu-D3b), one (spot 21) in 'Gabo' (Glu$D 3 b$ ), four (spots 3, 4, 5, and 6) in 'Aroona' (Glu-D3c), and four (spots 29, 30, 31, and 32) in 'Insignia' (Glu-D3c) to identify LMW-GS alleles at the Glu-3D locus (Figure 2 and Supplementary Figure S2). Four proteins (spots 3, 4, 5, and 6) in 'Aroona' (Glu-D3c) and four (spots 29, 30, 31, and 32) in 'Insignia' (Glu-D3c) were identified as LMW-GSs encoded by GluD3-32. Spot 12 from ARIL 36-2 (Glu-D3b) and spot 21 from 'Gabo' (Glu-D3b) were identified as LMW-GSs encoded by GluD3-31, indicating that the Glu-D3b and Glu-D3c alleles represent LMW-GSs encoded by GluD3-31 and GluD3-32, respectively (Table 2 and Tables S2 and S3).

\section{Discussion}

LMW-GSs are encoded by genes at Glu-3 loci (Glu-A3, Glu-B3, and Glu-D3), with copy numbers varying from 10-40. LMW-GS alleles at the Glu-3 loci determine end-use quality. To evaluate the dough-processing qualities of wheat cultivars, LMW-GS alleles at the Glu-3 loci have been ranked for dough strength in wheat cultivars from Australia [25] and New Zealand [29] and for the 'Aroona' near-isogenic lines (ARILs) [19,31]. Since the composition of LMW-GS alleles in individual cultivars has been given priority during wheat breeding, SDS-PAGE, 2-DGE, HPLC, and LMW-GS molecular marker systems have been used to define LMW-GS alleles in different wheat cultivars. Previously, one to two peaks corresponding to LMW-GSs encoded at each Glu-3 locus were identified by the composition analysis of LMW-GS in common wheat cultivars using a RP-UHPLC 
method [24]. In this study, two to five LMW-GS peaks in RP-HPLC corresponding to Glu$A 3,-B 3$, and -D3 alleles were determined through comparative analysis of 'Aroona' and the 12 ARILs. The accuracy was confirmed by comparing the resulting RP-HPLC patterns with patterns in standard wheat cultivars and suggests that our improved RP-HPLC method can be used to identify LMW-GS alleles faster, easier, and more accurately than traditional methods such as SDS-PAGE. However, three Glu-A3 alleles (Glu-A3a, $-A 3 b$, and $-A 3 c)$ and two Glu-D3 alleles (Glu-D3b and -D3f) could not be discriminated. Furthermore, by integrating our 2-DGE data on LMW-GSs in this study with the composition of LMW-GS genes previously characterized in 'Aroona' and ARILs [19,28,36], we created reference maps (Figure 3) for protein spots corresponding to each LMW-GS allele in three standard cultivars, 'Gabo' (Glu-A3b, -B3b, and -D3b), 'Aroona' (Glu-A3c,-B3b, and $-D 3 c)$, and 'Orca' $(G l u-A 3 d,-B 3 d$, and $-D 3 c)$.

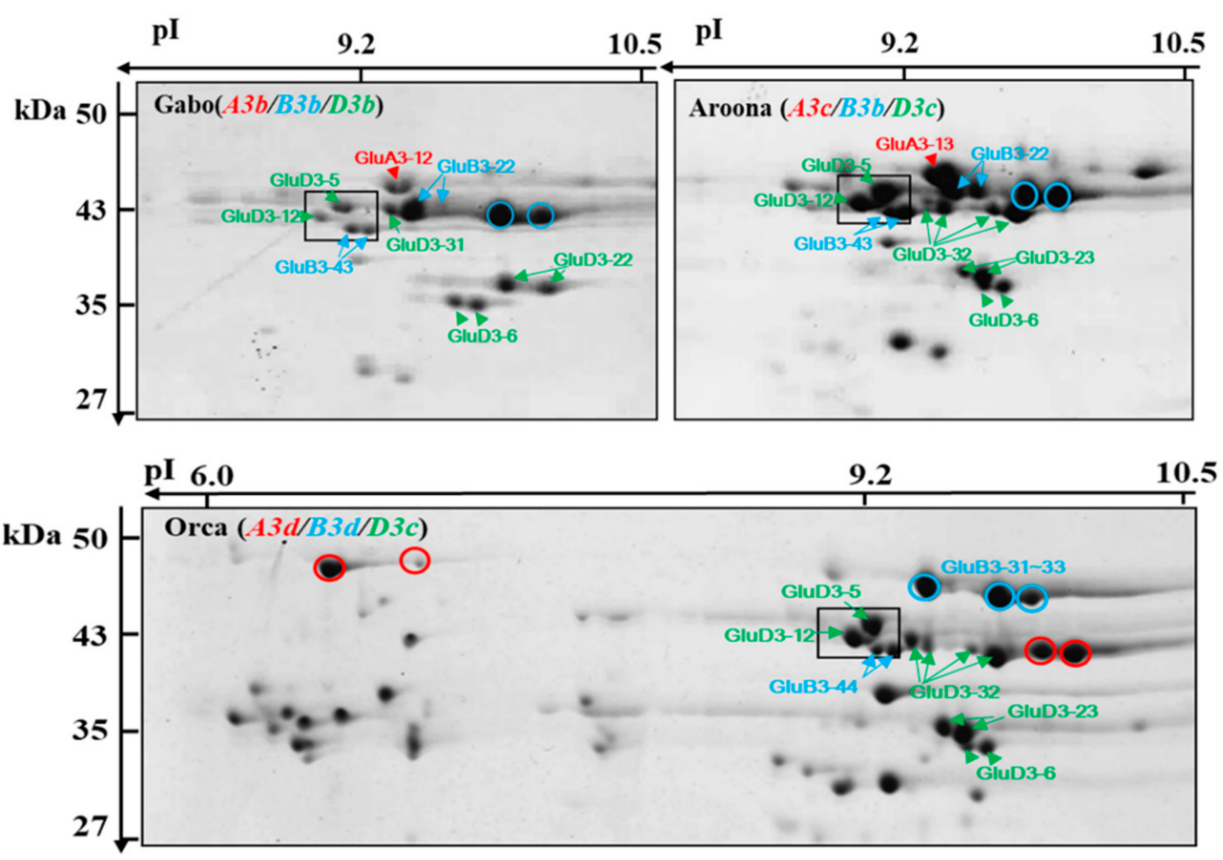

Figure 3. Two-dimensional gel electrophoresis (2-DGE) separation of LMW-GSs from three wheat cultivars: 'Chinese spring' (Glu-A3a/B3a/C3a), 'Gabo' (Glu-A3b/B3b/C3b), 'Aroona' (Glu-A3c/B3b/C3c), and 'Orca' $(G l u-A 3 d / B 3 d / C 3 c)$. Protein spots corresponding to each LMW-GS allele encoded by $G l u-A 3, G l u-B 3$, and Glu-C3 are indicated by red, light blue, and green colors, respectively, based on integrated comparative analysis and identification using MS/MS.

\subsection{Glu-A3 Alleles}

We identified 4-6 genes at the Glu-A3 locus in 'Aroona' (Glu-A3c) and Glu-A3 ARILs using an LMW-GS gene molecular marker system [19,31,37]. Except for ARIL18-5 (Glu-A3d) with three active LMW-GS genes (GluA3-4, GluA3-23, and A3-568), the other lines have one active gene: GluA3-11 in ARIL15-4 (Glu-A3a), GluA3-12 in ARIL16-1 (Glu-A3b), GluA3-13 in 'Aroona' (Glu-A3c), GluA3-15/17 in ARIL19-2 (Glu-A3e), and GluA3-16 in ARIL20-1 (Glu-A3f) as shown in Table 3. We also previously analyzed the composition of LMW-GS alleles in 32 Korean wheat cultivars and 11 standard wheat cultivars using 2-DGE and SDS-PAGE $[23,27]$. One LMW-i type subunit encoded by the GluA3-13 (A3-620) gene was identified in 'Jokyung' (Glu-A3c) and 'Cheyenne' (Glu-A3c). By comparing LMW-GS spot patterns with those from 'Jokyung' [27], we defined one LMW-i type subunit (red circle in Figure 1A) corresponding to GluA3-13 in 'Aroona' (Glu-A3c). 
Table 3. Active LMW-GS genes at the Glu-A3, Glu-B3, and Glu-D3 in 'Aroona' and 'Aroona' near-isogenic lines (ARILs).

\begin{tabular}{|c|c|c|c|c|c|c|c|}
\hline Line & Alleles & \multicolumn{6}{|c|}{ a Active LMW-GS Genes ( ${ }^{b}$ Gene Name in Chinese Wheat Germplasm) } \\
\hline \multicolumn{8}{|l|}{ At $G l u-3 A$} \\
\hline ARIL15-4 & Glu-A3a & & & $\begin{array}{l}\text { GluA3-11 } \\
(A 3-620)\end{array}$ & & & \\
\hline ARIL16-1 & $G l u-A 3 b$ & & & $\begin{array}{l}\text { GluA3-12 } \\
(A 3-643)\end{array}$ & & & \\
\hline Aroona & Glu- $A 3 c$ & & & $\begin{array}{l}\text { GluA3-13 } \\
(A 3-620)\end{array}$ & & & \\
\hline ARIL18-5 & Glu-A3d & $\begin{array}{l}\text { GluA3-23 } \\
(A 3-402)\end{array}$ & $\begin{array}{c}- \\
(A 3-568)\end{array}$ & $\begin{array}{l}\text { GluA3-4 } \\
(A 3-662)\end{array}$ & & & \\
\hline ARIL19-2 & Glu-A3e & & & $\begin{array}{c}\text { GluA3-15/17 } \\
(\text { A3-646) }\end{array}$ & & & \\
\hline ARIL20-1 & Glu-A3f & & $\begin{array}{c}\text { GluA3-16 } \\
(A-573)\end{array}$ & & & & \\
\hline \multicolumn{8}{|l|}{ At Glu-3B } \\
\hline ARIL21-2 & Glu-B3a & $\begin{array}{l}\text { GluB3-44 } \\
(B 3-530 a)\end{array}$ & $\begin{array}{c}\text { GluB3-11 } \\
(\text { B3-593) }\end{array}$ & $\begin{array}{l}\text { GluB3-21 } \\
(B 3-624)\end{array}$ & & & \\
\hline Aroona & Glu-B3b & $\begin{array}{l}\text { GluB3-43 } \\
(B 3-530 b)\end{array}$ & $\begin{array}{c}\text { GluB3-12 } \\
(B 3-607)\end{array}$ & $\begin{array}{l}\text { GluB3-22 } \\
(B 3-621 a)\end{array}$ & & & \\
\hline ARIL23-4 & Glu-B3c & $\begin{array}{l}\text { GluB3-44 } \\
(B 3-530 a)\end{array}$ & & $\begin{array}{l}\text { GluB3-34 } \\
(B 3-688 a)\end{array}$ & & & \\
\hline ARIL24-3 & Glu-B3d & $\begin{array}{l}\text { GluB3-44 } \\
\text { (B3-530a) }\end{array}$ & & $\begin{array}{c}\text { GluB3-31-33 } \\
(\text { B3-688b) }\end{array}$ & & & \\
\hline ARIL27-6 & Glu-B3g & $\begin{array}{l}\text { GluB3-41 } \\
(B 3-530 c)\end{array}$ & $\begin{array}{c}\text { GluB3-15 } \\
(\text { B3-544) }\end{array}$ & $\begin{array}{l}\text { GluB3-23 } \\
(B 3-621 b)\end{array}$ & & & \\
\hline ARIL28-4 & Glu-B3h & $\begin{array}{l}\text { GluB3-43 } \\
(B 3-530 b)\end{array}$ & & $\begin{array}{c}\text { GluB3-61-64 } \\
(B 3-688 c)\end{array}$ & & & \\
\hline \multicolumn{8}{|l|}{ At $G l u-3 D$} \\
\hline ARIL36-2 & Glu-D3b & $\begin{array}{l}\text { GluD3-6 } \\
\text { (D3-385) }\end{array}$ & $\begin{array}{l}\text { GluD3-4 } \\
\text { (D3-394) }\end{array}$ & $\begin{array}{l}\text { GluD3-22 } \\
\text { (D3-441) }\end{array}$ & $\begin{array}{l}\text { GluD3-12 } \\
\text { (D3-528) }\end{array}$ & $\begin{array}{l}\text { GluD3-5 } \\
\text { (D3-575) }\end{array}$ & $\begin{array}{l}\text { GluD3-31 } \\
(D 3-578 b)\end{array}$ \\
\hline Aroona & $G l u-D 3 c$ & $\begin{array}{l}\text { GluD3-6 } \\
\text { (D3-385) }\end{array}$ & $\begin{array}{l}\text { GluD3-4 } \\
\text { (D3-394) }\end{array}$ & $\begin{array}{l}\text { GluD3-23 } \\
\text { (D3-432) }\end{array}$ & $\begin{array}{l}\text { GluD3-12 } \\
\text { (D3-528) }\end{array}$ & $\begin{array}{l}\text { GluD3-5 } \\
\text { (D3-575) }\end{array}$ & $\begin{array}{l}\text { GluD3-32 } \\
\text { (D3-578a) }\end{array}$ \\
\hline ARIL35-1 & Glu-D3f & $\begin{array}{l}\text { GluD3-6 } \\
\text { (D3-385) }\end{array}$ & $\begin{array}{l}\text { GluD3-4 } \\
\text { (D3-394) }\end{array}$ & $\begin{array}{c}\text { GluD3-22 } \\
\text { (D3-441) }\end{array}$ & $\begin{array}{c}\text { GluD3-12 } \\
\text { (D3-528) }\end{array}$ & $\begin{array}{l}\text { GluD3-5 } \\
\text { (D3-575) }\end{array}$ & $\begin{array}{l}\text { GluD3-31 } \\
\text { (D3-578b) }\end{array}$ \\
\hline
\end{tabular}

a LMW-GS genes identified from Glu-A3 [37], Glu-B3 [34], and Glu-D3 [35] ${ }^{\mathrm{b}}$ LMW-GS genes isolated from 'Aroona', ARILs, and Chinese wheat germplasm $[19,36]$.

The retention times of LMW-GSs corresponding to Glu-A3 alleles in the RP-HPLC analysis (Figure 1A) and their spot patterns in 2-DGE (Figure 2A) were similar among ARIL 15-4 (Glu-A3a), ARIL 16-1 (Glu-A3b), and 'Aroona' (Glu-A3c). Similar results were observed among the standard cultivars 'Chinese Spring' (Glu-A3a), 'Gabo' (Glu-A3b), and 'Cheyenne' (Glu-A3c) (Supplementary Figures S1 and S2), indicating that it is difficult to discriminate LMW-GSs derived from Glu-A3a, Glu-A3b, and Glu-A3c alleles using either RP-HPLC or 2-DGE methods. In 'Aroona' near-isogenic lines, the Glu-A3a allele in ARIL 15-4 was donated by 'Chinese Spring', where one gene (GluA3-11) at Glu-A3a encoding an LMW-i type subunit is actively expressed [38]. The Glu-A3b allele in ARIL 16-1 comes from 'Gabo', where one gene (GluA3-12) at Glu-A3b encoding an LMW-i type subunit is expressed [19]. The LMW-GSs encoded by Glu-A3 alleles in ARIL 15-4 (Glu-A3a), ARIL 16-1 (Glu-A3b), and 'Aroona' (Glu-A3c) consist of 356, 364, and 356 amino acid residues 
excluding signal peptides, respectively. They contain ISQQQQ N-terminal sequences and have $99.44-99.72 \%$ identity to each other, resulting in difficulty in distinguishing them.

Through the comparative analysis of RP-HPLC patterns for LMW-GS fractions from ARIL 18-5 (Glu-A3d), ARIL 19-2 (Glu-A3e), and ARIL 20-1 (Glu-A3f), we identified five, three, and two unique peaks, respectively (Figure 1A). Four and one unique protein spots were observed in 2-DGE for ARIL18-5 (Glu-A3d) and ARIL20-1 (Glu-A3f), respectively, but no distinctly different spot was observed for ARIL19-2 (Glu-A3e). We observed similar results for the standard cultivars, 'Orca' (Glu-A3d), 'Neepawa' (Glu-A3e), and 'Clement (Glu-A3f), as shown in Figures S1 and S2. Using the LMW-GS gene molecular marker system, Zhang et al. (2012) [19] determined that the Glu-A3d allele represents three actively expressed genes, GluA3-4 (A3-662), GluA3-23 (A3-402), and (A3-568) and that the Glu-A3f allele corresponds to one expressed gene, GluA3-16 (A3-573), encoding an i-type LMW-GS with 336 amino acid residues, $39.04 \mathrm{kDa}$, and ISQQQQQP N-terminal. In this study, we observed one unique LMW-GS spot at about $39 \mathrm{kDa}$ in 2-DGE of ARIL 20-1 (Glu-A3f), as shown in Figure 2A, indicating that the spot could be derived from GluA3-16 (A3573). Moreover, ARIL 18-5 (Glu-A3d) possessed three actively expressed LMW-GS genes, encoding GluA3-23 (A3-402)/A3-568/ GluA3-4 (A3-662), in which the signal peptidetruncated forms have 284/338/370 amino acid residues, 32.22/39.28/43.02 kDa, and MDTSCIP/ISQQQQPP/ISQQQQQP N-terminal sequences, respectively. We observed four unique spots, two at $43 \mathrm{kDa}$ and two at $39 \mathrm{kDa}$, in the 2-DGE patterns of ARIL 18-5 $(G l u-A 3 d)$ compared to those of 'Aroona' (Glu-A3c), as shown in Figure 2A, indicating that these spots could be GluA3-4 (A3-662) and A3-568, respectively, based on predicted molecular weight and comparative analysis with 'Aroona' and the ARILs.

\subsection{Glu-B3 Alleles}

Active genes encoding m- and s-type LMW-GS genes at the Glu-B3 locus of 'Aroona' (Glu-B3b) and five ARILs (Glu-B3a, -B3c,-B3d, -B3g, and -B3h) were identified by using the LMW-GS gene molecular marker system $[19,34]$. The Glu-B3 alleles were divided into two groups according to the composition of s-type genes, one group comprising Glu-B3a,-B3b, and $-B 3 g$ and the other comprising Glu-B3c, $-B 3 d$, and $-B 3 h$. The Glu-B3a, $-B 3 b$, and $-B 3 g$ alleles correspond to three actively expressed LMW-GS genes, GluB3-11/-21/-44, GluB312/-22/-43, and GluB3-15/-23/-41, respectively, as shown in Table 3, while the Glu-B3c, $-B 3 d$, and -B3h alleles represent two actively expressed genes, GluB3-34/-44, GluB3-31-33/44, and GluB3-61-64/-43, respectively. Proteins encoded by LMW-GS genes in each group showed similar electrophoretic patterns in 2-DGE [19]. In this study, we also observed that LMW-GSs from ARIL 21-2 (Glu-B3a), 'Aroona' (Glu-B3b), and ARIL 27-6 (Glu-B3g) showed similar patterns in 2-DGE while those from ARIL 23-4 (Glu-B3c), ARIL 24-3 (Glu-B3d), and ARIL 28-4 (Glu-B3h) were similar (Figure 2B).

ARIL 28-4 (Glu-B3h) has two active genes: GluB3-43 (B3-530b) and GluB3-61-64 (B3688c) [19]. Previously, we reported that six protein spots were derived from the Glu-B3h allele in Korean wheat cultivar 'Jokyung' [27]. Of these six, two encoded by GluB3-43 were m-type LMW-GSs with a METSHIP N-terminal, corresponding to B3-530b, and four encoded by GluB3-33 were s-type LMW-GSs with a MENSHIP N-terminal, identical to B3-688b and sharing $98.92 \%$ identity with B3-688c. Thus, compared with 'Jokyung' (Glu$B 3 h$ ), two spots (light blue arrows in the positioning box in Figure 2B) from ARIL28-4 (Glu-B3h) represent m-type LMW-GSs encoded by GluB3-43 (B3-530b). The other four spots (light blue circles) could be identical to s-type LMW-GSs encoded by GluB3-61-64 (B3-688c). Furthermore, two spots corresponding to GluB3-43 (B3-530b), light blue arrows in the positioning box in Figure 2B, in ARIL28-4 (Glu-B3h) were observed in ARIL 21-2 (Glu-B3a), 'Aroona' (Glu-B3b), ARIL 23-4 (Glu-B3c), ARIL 24-3 (Glu-B3d), and ARIL 27-6 (Glu-B3g). These lines possess one of the GluB3-41/-43/-44 genes encoding an m-type LMW-GS consisting of 330 amino acids, which have $99.09-99.39 \%$ identity to each other. These results indicate that two spots (light blue arrows) in a square box in Figure 2B are derived from a gene encoding B3-530. 
ARIL 27-6 (Glu-B3g) shared four spots (light blue circles) with 'Aroona' (Glu-B3b) and had three unique spots (Figure 2B). The spot patterns were similar to those of their standard cultivars, 'Gabo' (Glu-B3b) and 'Glenlea' (Glu-B3g), respectively. Three unique proteins (spots 9, 10, and 11) in ARIL 27-6 (Glu-B3g) and three (spots 26, 27, and 28) in 'Glenlea' (Glu-B3g) were identified as s-type LMW-GSs (ABY58126 or ACZ59817) encoded by the GluB3-15 (B3-544) gene (Table 2 and Supplementary Table S2). In ARIL 27-6 (Glu-B3g), three LMW-GS genes have been reported to be actively expressed, GluB3-15 (B3-544), GluB3-23 (B3-621), and GluB3-41 (B3-530c) [19], indicating that the four common spots at $42 \mathrm{kDa}$ in the 2-DGE of ARIL 27-6 (Glu-B3g) and 'Aroona' (Glu-B3b) could be derived from a gene encoding B3-621. Indeed, we identified two proteins (spots 1 and 2 ) in the 2-DGE of 'Aroona' (Glu-B3b) and two (spots 22, and 23) in 'Gabo' (Glu-B3b) as s-type LMW-GSs encoded by the GluB3-22 (B3-621a) gene haplotype (Table 2 and Supplementary Table S2). The two LMW-GSs (blue circles in Figure 2B) in ARIL 21-2 (Glu-B3a), four in 'Aroona' (GluB3b), and four in ARIL 27-6 (Glu-B3g) could be GluB3-21 (B3-624), GluB3-21 (B3-621a), and GluB3-23 (B3-621b), respectively; however, the spots in the 2-DGE of ARIL 21-2 (Glu-B3a) and 'Aroona' (Glu-B3b) could also be derived from the GluB3-11 (B3-593) and GluB3-12 (B3-607) genes, respectively.

ARIL 23-4 (Glu-B3c), ARIL 24-3 (Glu-B3d), and ARIL 28-4 (Glu-B3h) have two active genes, GluB-34/-44, GluB-31-33/-44, and GluB-61-64/-43, respectively. These lines and their standard cultivars showed similar protein spot patterns in 2-DGE (Figure 2B, and Figure S1). Compared to 'Aroona' (Glu-B3b), ARIL 23-4 (Glu-B3c), ARIL 24-3 (Glu-B3d), and ARIL 28-4 (Glu-B3h) had two common spots (light blue arrows within black square in Figure 2B) encoded by one of the GluB-41/-44 genes. ARIL 23-4 (Glu-B3c), ARIL 24-3 $(G l u-B 3 d)$, and ARIL 28-4 (Glu-B3h) contain two, three, and four unique proteins, respectively, indicating that these could be encoded by GluB-34, GluB-31-33, and GluB-61-64, respectively.

\subsection{Glu-D3 Alleles}

At the Glu-D3 locus, six active genes were identified in ARIL 36-2 (Glu-D3b), Aroona (GluD3c), and ARIL 35-1 (Glu-D3f), including GluD3-6 (D3-385), GluD3-4 (D3-394), GluD3-22/-23 (D3-441/-432), GluD3-12 (D3-528), GluD3-5 (D3-575), and GluD3-31/-32 (D3-578b/a) [19,35]. We previously identified eight LMW-GS protein spots encoded by five gene haplotypes using 2-DGE in 'Jokyung' (Glu-D3a) and 'Keumkang' (Glu-D3a): GluD3-6 (D3-385), GluD3-21 (D3441), GluD3-11 (D3-525), GluD3-5 (D3-575), and GluD3-31 (D3-578b); LMW-GS encoded by GluD3-4 (D3-394) was not identified due to an insufficient amount of protein [27,28]. Moreover, 'Aroona' (Glu-D3c) is known to contain six active LMW-GS genes: GluD3-6 (D3-385), GluD3-4 (D3-394), GluD3-23 (D3-432), GluD3-12 (D3-528), GluD3-5 (D3-575), and GluD3-32 (D3-578a). In this study, we found 10 protein spots encoded by LMW-GS genes at the Glu-D3c locus in 'Aroona'. Four of these were shown to have similar positions on 2-DGE gels compared to those identified in 'Jokyung' (Glu-D3a) and 'Keumkang' (Glu-D3a): GluD3-6 (D3-385), GluD3-11 (D3-525), and GluD3-5 (D3-575). We identified four protein spots (spot 3, 4, 5, and 6) in 'Aroona' (Glu-D3c) and four (spot 29, 30, 31, and 32) in 'Insignia' (Glu-D3c) encoded by the GluD3-32 (D3-578a) haplotype. Although the remaining two spots were not identified by MS/MS analysis, considering that GluD3-4 (D3-394) is insufficient amount for our 2-DGE system [27,28], we predicted them to be GluD3-23 (D3-432) based on the genetic marker system and comparative analysis.

ARIL 36-2 (Glu-D3b) and ARIL 35-1 (Glu-D3f) have six active LMW-GS genes such as GluD3-6 (D3-385), GluD3-4 (D3-394), GluD3-22 (D3-441), GluD3-12 (D3-528), GluD35 (D3-575), and GluD3-31 (D3-578b). These ARILs and their corresponding standard wheat cultivars, 'Gabo' (Glu-D3b) and 'Cheyenne' (Glu-D3f), showed similar protein spot patterns in 2-DGE. Compared with those in 'Aroona' (Glu-D3c) and 'Insignia' (Glu-D3c), we observed two unique protein spots at $35 \mathrm{kDa}$ and one at $43 \mathrm{kDa}$ between 9.2 and $10.5 \mathrm{pI}$ in 2-DGE of ARIL36-2 (Glu-D3b) and ARIL35-1 (Glu-D3f) in Figure 2C. The two spots at $35 \mathrm{kDa}$ were previously observed in 2-DGE of 'Jokyung' (Glu-D3a) and are encoded by the 
GluD3-21 (D3-441) haplotype. The other spot at $43 \mathrm{kDa}$, spot 12 for ARIL 36-2 (Glu-D3b) and spot 21 for 'Gabo' (Glu-D3b), was identified as a LMW-GS encoded by the GluD3-31 (D3-578b) haplotype.

Finally, based on an integrative analysis between the activated LMW-GS genes and 2DGE separation of LMW-GSs in 'Aroona' and its ARILs, we tried to designate the activated genes corresponding to LMW-GS spots separated on 2-DGE in 'Aroona' (Glu-A3c/-B3b/$\mathrm{C} 3 c$ ) and two standard cultivars, 'Gabo' (Glu-A3b/-B3b/-C3b) and 'Orca' (Glu-A3d/-B3d/C3c), as shown in Figure 3. The results might be used to identify LMW-GS alleles in germplasm prior to breeding and to screen for desirable LMW-GS alleles in wheat quality improvement.

\section{Materials and Methods}

\subsection{Plant Materials}

'Aroona' and its 12 near-isogenic lines differing at the Glu-A3, Glu-B3, and Glu-D3 loci (Table 1) were provided by Dr. Marie Appelbee and Prof. Ken Shepherd, SARDI Grain Quality Research Laboratory, South Australia. The grains of 10 standard wheat cultivars used for the identification of Glu-3 alleles (Supplementary Table S1) were kindly provided by the National Bioresource Project-Wheat, Japan (NBRP-Wheat, https:/ / shigen.nig.ac.jp/ wheat/komugi/, 12 October 2015) and by the National Plant Germplasm System of the USDA-ARS, USA (NPGS, https:/ / www.ars-grin.gov/npgs/, 12 October 2015). The grains of the wheat cultivars were ground with a mortar and pestle to fine powders and stored at $-80^{\circ} \mathrm{C}$ until glutenin extraction.

\subsection{Glutenin Extraction}

Glutenin was extracted as previously reported [23]. Wheat flour (300 mg) was mixed with $15 \mathrm{~mL}$ of $50 \%$ propanol $(v / v)$ at $65^{\circ} \mathrm{C}$ for $30 \mathrm{~min}$ and then centrifuged at $10,000 \times g$ for $5 \mathrm{~min}$. The supernatant fraction containing gliadin was removed. This extraction was repeated three times to minimize gliadin contamination. The precipitate was suspended in $1.5 \mathrm{~mL}$ of $50 \%(v / v)$ propanol, $80 \mathrm{mM}$ Tris- $\mathrm{HCl}(\mathrm{pH} 8.0)$, and $1 \%(w / v)$ dithiothreitol (DTT) at $65^{\circ} \mathrm{C}$ for $30 \mathrm{~min}$. After centrifugation at $10,000 \times \mathrm{g}$ for $5 \mathrm{~min}, 1.5 \mathrm{~mL}$ of $80 \mathrm{mM}$ Tris- $\mathrm{HCl}$ ( $\mathrm{pH} 8.0$ ) with $1.4 \%$ 4-vinylpyridine $(v / v)$ was added for alkylation and incubated at $65^{\circ} \mathrm{C}$ for $15 \mathrm{~min}$. After centrifugation at $10,000 \times g$ for $2 \mathrm{~min}$, the supernatant was transferred to a new $1.5 \mathrm{~mL}$ tube and stored at $-4{ }^{\circ} \mathrm{C}$ overnight. Glutenin fractions were precipitated using acetone containing $15 \%$ TCA $(v / v)$ and stored at $-20{ }^{\circ} \mathrm{C}$ until use.

\subsection{Two-Dimensional Gel Electrophoresis (2-DGE)}

For 2-DGE, precipitated glutenin samples were washed with chilled acetone, centrifuged at $12,000 \times g$ for $10 \mathrm{~min}$, and then dried. The glutenin pellets were dissolved completely using $70 \mu \mathrm{L}$ of dehydration buffer (7 M urea, $2 \mathrm{M}$ thiourea, $2 \%(w / v)$ CHAPS, and $0.5 \%(v / v)$ IPG buffer (GE Healthcare Life Sciences, USA) and $12.8 \mu \mathrm{L}$ of $1 \mathrm{M}$ DTT. Total protein was quantified using the Bradford method [39]. Fifty micrograms of glutenin was resolved in $350 \mu \mathrm{L}$ rehydration solution containing $12.8 \mu \mathrm{L}$ of $1 \mathrm{M}$ DTT, applied on an $18 \mathrm{~cm}$ IPG strip (pH 6-11, GE Healthcare Life Sciences, USA) and then rehydrated in-gel for a total run time of $80 \mathrm{kVh}$ at $20^{\circ} \mathrm{C}$ using the IPGphore system (Amersham Biosciences, GE Healthcare Life Sciences, USA). The IPG strips were equilibrated using $6 \mathrm{M}$ urea, $75 \mathrm{mM}$ Tris- $\mathrm{HCl}(\mathrm{pH} 8.8), 29.3 \%(v / v)$ glycerol, $2 \%(w / v)$ SDS, and 1\% $(w / v)$ DTT for $15 \mathrm{~min}$ and then incubated with $6 \mathrm{M}$ urea, $75 \mathrm{mM}$ Tris- $\mathrm{HCl}(\mathrm{pH} 8.8), 29.3 \%(v / v)$ glycerol, $2 \%(w / v)$ SDS, and $2.5 \%(w / v)$ iodoacetamide for $15 \mathrm{~min}$. The 2-DGE (12.5\% acrylamide gel) was carried out at $70 \mathrm{~V}$ for $17 \mathrm{~h}$ and at $130 \mathrm{~V}$ for $6 \mathrm{~h}$. The gel was stained with Coomassie Brilliant Blue R-250 for $3 \mathrm{~h}$, de-stained with a water-methanol-acetic acid buffer (80/10/10, by volume) twice for $3 \mathrm{~h}$ each, and then scanned using an Epson Perfection V800 Photo scanner (Epson, Japan). Finally, the patterns of protein spots separated on the gels were analyzed using Image Master Platinum v6.0 (GE Healthcare Life Sciences, Piscataway, NJ, USA). 


\subsection{Identification of LMW-GSs Using UPLC-MS/MS}

Protein spots from 'Aroona' and 12 ARILs were excised from 2-D gels; reduced; alkylated; digested with trypsin, chymotrypsin, and thermolysin; and then analyzed as previously described [40]. The protein spots from 10 standard wheat cultivars were excised from 2-D gels and digested in-gel with chymotrypsin. The digested peptides were subjected to nanoAcquity UPLC coupled with MS (Waters Synapt G1 HDMS, MA, USA) to obtain their mass spectra. The data were analyzed using a database containing 63,245 Triticum protein sequences from NCBI (www.ncbi.nlm.nih.gov, 12 October 2015) and 151,173 Triticum protein sequences from UniProt (www.uniprot.org, 12 October 2015). UPLC-MS/MS analysis was conducted at the NICEM (National Instrumentation Center for Environmental Management, Seoul National University, Korea).

\subsection{Separation of LMW-GSs Using RP-HPLC}

An analysis of the LMW-GSs using RP-HPLC was performed using a Waters Alliance e2695 (USA) equipped with an XBridge Protein BEH-C4 column $(3.5 \mathrm{~m}, 4.6 \times 250 \mathrm{~nm}$ i.d., Waters, USA) following the method of Yu et al. (2013) with modifications [24]. The precipitated glutenin samples were completely dissolved in $500 \mu \mathrm{L}$ of $0.1 \%(v / v)$ trifluoroacetic acid with $20 \%(v / v)$ acetonitrile and filtered using a PVDF syringe filter $(0.45 \mathrm{~m}$, Whatman, Maidstone, UK). Ten microliters of each sample were subjected to RP-HPLC with the following solvents: water with $0.1 \%$ trifluoroacetic acid (solvent $\mathrm{A}$ ) and $20 \%(v / v)$ acetonitrile with $0.1 \%$ trifluoroacetic acid (solvent $\mathrm{B}$ ). The proteins were eluted using a linear gradient from 25 to $45 \%$ of solvent B for $50 \mathrm{~min}$ under a $0.8 \mathrm{~mL} / \mathrm{min}$ flow rate and $65{ }^{\circ} \mathrm{C}$ column oven temperature and monitored at $206 \mathrm{~nm}$ wavelength.

Supplementary Materials: The following are available online at https: / www.mdpi.com/article/10 $.3390 /$ ijms22147709/s1, Figure S1: RP-HPLC analysis of LMW-GS fractions in 10 standard wheat cultivars, 'Chinese spring' (Glu-A3a/B3a), 'Gabo' (Glu-A3b/B3b/D3b), 'Cheyenne' (Glu-A3c/D3f), 'Neepawa' (Glu-A3e), 'Clement' (Glu-A3f), 'Halberd' (Glu-B3c), 'Orca' (Glu-A3d/B3d), 'Suneca' (Glu$B 3 h)$, 'Insignia' (Glu-D3c), and 'Glenlea' (Glu-B3g). Peaks corresponding to each LMW-GS allele encoded by Glu-A3, Glu-B3, and Glu-C3 are indicated by red, light blue, and green arrowheads, respectively. Figure S2: Two-DGE analysis of LMW-GSs from 10 standard wheat cultivars: 'Chinese spring' (Glu-A3a/B3a), 'Gabo' (Glu-A3b/B3b/D3b), 'Cheyenne' (Glu-A3c/D3f), 'Neepawa' (Glu-A3e), 'Clement' (Glu-A3f), 'Halberd' (Glu-B3c), 'Orca' (Glu-A3d/B3d), 'Suneca' (Glu-B3h), 'Insignia' (Glu$D 3 c)$, and 'Glenlea' (Glu-B3g). Protein spots corresponding to each LMW-GS allele encoded by Glu-A3, $\mathrm{Glu}-\mathrm{B3}$, and $\mathrm{Glu}-\mathrm{C} 3$ are indicated by red, light blue, and green arrows, respectively. Numbered spots were identified using LC-MS/MS. Table S1: Standard wheat cultivars used to distinguish alleles at the Glu-A3, Glu-B3, Glu-D3 loci. Table S2: Identification of 2-DGE spots in LMW-GS fractions from flour of 'Aroona', ARILs, and standard wheat cultivars by MS/MS.

Author Contributions: Conceptualization, K.C., S.-H.L. and J.-Y.L.; formal analysis, K.C., Y.-R.J., S.B.A., Y.Q.G. and A.S.-B.; funding acquisition, K.C. and J.-Y.L.; investigation, Y.-R.J., S.-H.L., S.B.A., Y.Q.G., A.S.-B. and J.-Y.L.; methodology, Y.-R.J., S.-H.L., S.B.A., Y.Q.G., A.S.-B. and J.-Y.L.; project administration, J.-Y.L.; supervision, J.-Y.L.; validation, S.B.A. and J.-Y.L.; visualization, K.C. and Y.-R.J.; writing—original draft, K.C. and J.-Y.L.; writing—review and editing, K.C., S.B.A., Y.Q.G. and J.-Y.L. All authors have read and agreed to the published version of the manuscript.

Funding: This work was supported by grants from the National Institute of Agricultural Science (RDA PJ014838), Rural Development Administration, Korea. This study was supported by Basic Science Research Program through the National Research Foundation of Korea (NRF) funded by the Ministry of Education (2020R1I1A1A01069882) to K.C.

Institutional Review Board Statement: Not applicable.

Informed Consent Statement: Not applicable.

Acknowledgments: We thank Marie Appelbee and Ken Shepherd, SARDI Grain Quality Research Laboratory, Adelaide, South Australia, for the seeds of the spring wheat cultivar 'Aroona' and near-isogenic derivatives. 
Conflicts of Interest: The authors declare no conflict of interest.

\section{References}

1. Shewry, P.R.; Tatham, A.S.; Barro, F.; Barcelo, P.; Lazzeri, P. Biotechnology of breadmaking: Unraveling and manipulating the multi-protein gluten complex. Biotechnology 1995, 13, 1185-1190. [CrossRef]

2. Weegels, P.L.; Pijpekamp, A.M.; Graveland, A.; Hamer, R.J.; Schofield, J.D. Depolymerisation and re-polymerisation of wheat glutenin during dough processing. 1. Relationships between glutenin macropolymer content and quality parameters. J. Cereal Sci. 1996, 23, 103-111. [CrossRef]

3. Payne, P.I. Genetics of wheat storage proteins and the effect of allelic variation on bread-making quality. Annu. Rev. Plant Physiol. 1987, 38, 141-153. [CrossRef]

4. Wieser, H. Chemistry of gluten proteins. Food Microbiol. 2007, 24, 115-119. [CrossRef] [PubMed]

5. Wrigley, C.W. Giant proteins with flour power. Nature 1996, 381, 738-739. [CrossRef] [PubMed]

6. Payne, P.I.; Law, C.N.; Mudd, E.E. Control by homoeologous group 1 chromosomes of the high-molecular-weight subunits of glutenin, a major protein of wheat endosperm. Theor. Appl. Genet. 1980, 58, 113-120. [CrossRef]

7. Jackson, E.A.; Morel, M.H.; Sontag Strohm, T.; Branlard, G.; Metakovsky, E.; Redaelli, R. Proposal for combining the classification systems of alleles of Gli-1 and Glu-3 loci in bread wheat (Triticum aestivum L.). J. Genet. Breed. 1996, 50, 321-336.

8. Nagamine, T.; Kai, Y.; Takayama, T.; Yanagisawa, T.; Taya, S. Allelic variation at the Glu-1 and Glu-3 loci in southern Japanese wheats, and its effects on gluten properties. J. Cereal Sci. 2000, 32, 129-135. [CrossRef]

9. D'Ovidio, R.; Masci, S. The low-molecular-weight glutenin subunits of wheat gluten. J. Cereal Sci. 2004, 39, 321-339. [CrossRef]

10. Gupta, R.B.; Singh, N.K.; Shepherd, K. The cumulative effect of allelic variation in LMW and HMW glutenin subunits on dough properties in the progeny of two bread wheats. Theor. Appl. Genet. 1989, 77, 57-64. [CrossRef]

11. Cornish, G.B.; Bekes, F.; Allen, H.; Martin, D. Flour proteins linked to quality traits in an Australian doubled haploid wheat population. Aust. J. Agric. Res. 2001, 52, 1339-1348. [CrossRef]

12. Gianibelli, M.; Larroque, O.; MacRitchie, F.; Wrigley, C. Biochemical, genetic, and molecular characterization of wheat endosperm proteins. Cereal Chem. 2001, 78, 635-646. [CrossRef]

13. Wieser, H.; Kieffer, R. Correlations of the amount of gluten protein types to the technological properties of wheat flours determined on a micro-scale. J. Cereal Sci. 2001, 34, 19-27. [CrossRef]

14. Shewry, P.R.; Halford, N.G.; Tatham, A.S.; Popineau, Y.; Lafiandra, D.; Belton, P.S. The high molecular weightsubunitsofwheatgluteninandtheirroleindetermining wheat processing properties. Adv. Food Nutr. Res. 2003, 45, 219-302.

15. Payne, P.; Holt, L.; Jarvis, M.; Jackson, E. Two-dimensional fractionation of the endosperm proteins of bread wheat. Cereal Chem. 1985, 62, 319-326

16. Masci, S.; Rovelli, L.; Kasarda, D.; Vensel, W.; Lafiandra, D. Characterisation and chromosomal localisation of C-type lowmolecular-weight glutenin subunits in the bread wheat cultivar Chinese Spring. Theor. Appl. Genet. 2002, 104, 422-428. [CrossRef]

17. Ruiz, M.; Carrillo, J. Linkage relationships between prolamin genes on chromosomes 1A and 1B of durum wheat. Theor. Appl. Genet. 1993, 87, 353-360. [CrossRef]

18. Liu, C.-Y.; Shepherd, K. Inheritance of B subunits of glutenin and $\omega$-and $\gamma$-gliadins in tetraploid wheats. Theor. Appl. Genet. 1995, 90, 1149-1157. [CrossRef] [PubMed]

19. Zhang, X.; Jin, H.; Zhang, Y.; Liu, D.; Li, G.; Xia, X.; He, Z.; Zhang, A. Composition and functional analysis of low-molecular-weight glutenin alleles with Aroona near-isogenic lines of bread wheat. BMC Plant. Biol. 2012, 12, 243. [CrossRef]

20. Dong, K.; Hao, C.; Wang, A.; Cai, M.; Yan, Y. Characterization of HMW glutenin subunits in bread and tetraploid wheats by reversed-phase high-performance liquid chromatography. Cereal Res. Commun. 2009, 37, 65-73. [CrossRef]

21. Gao, L.; Ma, W.; Chen, J.; Wang, K.; Li, J.; Wang, S.; Bekes, F.; Appels, R.; Tan, Y. Characterization and comparative analysis of wheat high molecular weight glutenin subunits by SDS-PAGE, RP-HPLC, HPCE, and MALDI-TOF-MS. J. Agric. Food Chem. 2010, 58, 2777-2786. [CrossRef]

22. Liu, L.; Ikeda, T.M.; Branlard, G.; Peña, R.J.; Rogers, W.J.; Lerner, S.E.; Kolman, M.A.; Xia, X.; Wang, L.; Ma, W.; et al. Comparison of low molecular weight glutenin subunits identified by SDS-PAGE, 2-DE, MALDI-TOF-MS and PCR in common wheat. BMC Plant. Biol. 2010, 10, 124. [CrossRef]

23. Lee, J.Y.; Jang, Y.R.; Beom, H.R.; Altenbach, S.B.; Lim, S.H.; Lee, C.K. Allelic analysis of low molecular weight glutenin subunits using 2-DGE in Korean wheat cultivars. Breed. Sci. 2017, 67, 398-407. [CrossRef]

24. Yu, Z.; Han, C.; Yan, X.; Li, X.; Jiang, G.; Yan, Y. Rapid characterization of wheat low molecular weight glutenin subunits by ultraperformance liquid chromatography (UPLC). J. Agric. Food Chem. 2013, 61, 4026-4034. [CrossRef] [PubMed]

25. Gupta, R.B.; Shepherd, K. Two-step one-dimensional SDS-PAGE analysis of LMW subunits of glutelin. Theor. Appl. Genet. 1990, 80, 65-74. [CrossRef] [PubMed]

26. Dong, L.; Zhang, X.; Liu, D.; Fan, H.; Sun, J.; Zhang, Z.; Qin, H.; Li, B.; Hao, S.; Li, Z.; et al. New insights into the organization, recombination, expression and functional mechanism of low molecular weight glutenin subunit genes in bread wheat. PLoS ONE 2010, 5, e13548. [CrossRef] [PubMed]

27. Beom, H.-R.; Kim, J.S.; Jang, Y.-R.; Lim, S.-H.; Kim, C.-K.; Lee, C.K.; Lee, J.-Y. Proteomic analysis of low-molecular-weight glutenin subunits and relationship with their genes in a common wheat variety. 3 Biotech. 2018, 8, 56. [CrossRef] [PubMed] 
28. Lee, J.Y.; Beom, H.R.; Altenbach, S.B.; Lim, S.H.; Kim, Y.T.; Kang, C.S.; Yoon, U.-H.; Gupta, R.; Kim, S.-T.; Anh, S.-N.; et al. Comprehensive identification of LMW-GS genes and their protein products in a common wheat variety. Funct. Integr. Genom. 2016, 16, 269-279. [CrossRef]

29. Luo, C.; Griffin, W.; Branlard, G.; McNeil, D. Comparison of low-and high molecular-weight wheat glutenin allele effects on flour quality. Theor. Appl. Genet. 2001, 102, 1088-1098. [CrossRef]

30. Branlard, G.; Dardevet, M.; Saccomano, R.; Lagoutte, F.; Gourdon, J. Genetic diversity of wheat storage proteins and bread wheat quality. Euphytica 2001, 119, 59-67. [CrossRef]

31. Rasheed, A.; Xia, X.; Yan, Y.; Appels, R.; Mahmood, T.; He, Z. Wheat seed storage proteins: Advances in molecular genetics, diversity and breeding applications. J. Cereal Sci. 2014, 60, 11-24. [CrossRef]

32. Jin, H.; Zhang, Y.; Li, G.; Mu, P.; Fan, Z.; Xia, X.; He, Z. Effects of allelic variation of HMW-GS and LMW-GS on mixograph properties and Chinese noodle and steamed bread qualities in a set of Aroona near-isogenic wheat lines. J. Cereal Sci. 2013, 57, 146-152. [CrossRef]

33. Jang, Y.R.; Beom, H.R.; Altenbach, S.B.; Lee, M.K.; Lim, S.H.; Lee, J.Y. Improved Method for Reliable HMW-GS Identification by RP-HPLC and SDS-PAGE in Common Wheat Cultivars. Molecules 2017, 22, 1055. [CrossRef]

34. Wang, L.H.; Zhao, X.L.; He, Z.H.; Ma, W.; Appels, R.; Pena, R.J.; Xia, X.C. Characterization of low-molecular-weight glutenin subunit Glu-B3 genes and development of STS markers in common wheat (Triticum aestivum L.). Theor. Appl. Genet. 2009, 118, 525-539. [CrossRef] [PubMed]

35. Zhao, X.L.; Xia, X.C.; He, Z.H.; Lei, Z.S.; Appels, R.; Yang, Y.; Sun, Y.Y.; Ma, W. Novel DNA variations to characterize low molecular weight glutenin Glu-D3 genes and develop STS markers in common wheat. Theor. Appl. Genet. 2007, 114, 451-460. [CrossRef]

36. Zhang, X.; Liu, D.; Zhang, J.; Jiang, W.; Luo, G.; Yang, W.; Sun, J.; Tong, Y.; Cui, D.; Zhang, A. Novel insights into the composition, variation, organization, and expression of the low-molecular-weight glutenin subunit gene family in common wheat. J. Exp. Bot. 2013, 64, 2027-2040. [CrossRef]

37. Wang, L.H.; Li, G.Y.; Pena, R.J.; Xia, X.C.; He, Z.H. Development of STS markers and establishment of multiplex PCR for Glu-A3 alleles in common wheat (Triticum aestivum L.). J. Cereal Sci. 2010, 51, 305-312. [CrossRef]

38. Zhen, S.; Han, C.; Ma, C.; Gu, A.; Zhang, M.; Shen, X.; Li, X.; Yan, Y. Deletion of the low-molecular-weight glutenin subunit allele Glu-A3a of wheat (Triticum aestivum L.) significantly reduces dough strength and breadmaking quality. BMC Plant Biol. 2014, 14, 367. [CrossRef]

39. Bradford, M.M. A rapid and sensitive method for the quantitation of microgram quantities of protein utilizing the principle of protein-dye binding. Anal. Biochem. 1976, 72, 248-254. [CrossRef]

40. Altenbach, S.B.; Chang, H.-C.; Simon-Buss, A.; Mohr, T.; Huo, N.; Gu, Y.Q. Exploiting the reference genome sequence of hexaploid wheat: A proteomic study of flour proteins from the cultivar Chinese Spring. Funct. Integr. Genom. 2020, 20, 1-16. [CrossRef] 